\title{
As baterias motoras MABC-2, TGMD-2 e KTK explicam a função executiva em crianças de 8 a 10 anos?
}

https://doi.org/10.11606/issn.1981-4690.v35i2p343-352

\author{
Fernanda Cerveira Fronza* \\ Pâmella de Medeiros* \\ Elisa Pinheiro Ferrari** \\ Paola Cidade Cordeiro* \\ Kamylla Thais Dias de Freitas* \\ Fernando Luiz Cardoso*
}

\section{Resumo}

Objetivo deste estudo foi verificar se há relação entre o desempenho motor e as funções executivas, bem como, analisar qual bateria motora, MABC-2, KTK, TGMD-2, explica melhor as funções executivas de crianças de 8 a 10 anos com desenvolvimento típico. Fizeram parte da investigação 60 crianças, com idades entre oito e 10 anos (Média=9,09; desvio padrão 0,75). Para avaliação das funções executivas utilizou-se o Teste de Trilhas, já para avaliação do desempenho motor, utilizou-se os testes Movement Assessment Battery for Children Second Edition (MABC -2), Körperkoordination Test fürKinder (KTK) e Test of Gross Development Second Edition (TGMD-2). No que se refere às análises estatísticas, utilizou-se o teste de Correlação de Spearman para verificar a relação entre as funções executivas e o desempenho motor, e a Regressão linear simples foi utilizada para identificar quais os testes motores melhor explicam as funções executivas. Encontrou-se relação entre o desempenho motor e função executiva apenas para as meninas. Além disso, verificou-se que a bateria MABC-2 é o teste que melhor explica as funções executivas principalmente para o sexo feminino. Esses resultados implicam em uma possível detecção prévia parcial de dificuldades tanto motoras como cognitivas, por meio da bateria MABC-2 para o sexo feminino nesse grupo estudado. Ao mesmo tempo, com esses resultados, presume-se que apenas algumas tarefas motoras estão relacionadas à função executiva, sendo aquelas que envolvem habilidades motoras finas.

Palavras-chave: Destreza motora; Controle executivo; Baterias motoras; Desenvolvimento típico.

\section{Introdução}

A função executiva é um termo abrangente, corresponde a progressos cognitivos complexos, altamente ordenados e necessários para executar tarefas desafiadoras direcionadas a objetivos ${ }^{1}$. Correspondem a um sistema que funciona de maneira integrada, permitindo ao indivíduo tomar decisóes, avaliar e adequar seus comportamentos e estratégias ${ }^{2,3}$, favorecendo o planejamento e a resolução de problemas imediatos de médio a longo prazo e deste modo, fazer progressos em direção a metas ou objetivos ${ }^{3}$.

Especificamente, refere-se à memória de trabalho, atenção executiva, flexibilização mental e à inibição, sendo essas capacidades essenciais para o sucesso escolar, bem como desenvolvimento cognitivo, psicológico e motor ${ }^{2}$. Dessa maneira, as funções executivas estão altamente atreladas ao desempenho motor, principalmente no que tange o planejamento para execução de tarefas motoras, bem como, o monitoramento e a correção dos movimentos 5 .

A compreensão de que existe uma relação entre o desenvolvimento motor e o cognitivo têm sido amplamente discutidas na literatura ${ }^{6-10}$. No estudo de Houwen et al. ${ }^{10}$, os autores elucidaram que crianças de 3 a 5 anos com desordem coordenativa desenvolvimental apresentaram 
desempenho significativamente pior nas funçóes executivas, especificamente na memória de trabalho e planejamento/organização no cotidiano. Em complemento, relaçôes entre o domínio motor e cognitivo foram encontradas, dessa maneira os autores sugerem que programas complexos de intervenção motora podem ser utilizados para estimular habilidades cognitivas em crianças pré-púberes ${ }^{7}$.

Considerando essas associaçóes encontradas na literatura, defende-se que ao observar um desempenho motor baixo em crianças, pode ser viável alertar-se para possíveis problemas no campo cognitivo, como atenção, flexibilidade cognitiva e memória de trabalho ${ }^{11}$. Embora se tenha diversas baterias motoras mundialmente utilizadas (MABC-2, TGMD-2 e KTK), cada uma com as suas especificidades, o corpo de evidência que

\section{Método}

\section{Caracterização do estudo}

Este estudo descritivo transversal faz parte de um projeto maior intitulado "Exergames: estudo dos aspectos motores e cognitivos em ambientes escolares" o qual foi aprovado pelo Comitê de Ética em Pesquisa com Seres humanos da Universidade do Estado de Santa Catarina (CEPSH/UDESC) sob o número de processo $1.478 .305 / 2016$.

\section{Participantes}

Fizeram parte da investigação 60 crianças, selecionadas de forma não probabilística, considerando a disponibilidade das mesmas em fazer parte do estudo. Destas, 30 eram do sexo masculino com idades entre oito e 10 anos $(X=9,09 \pm 0,75)$, estudantes do $3^{\circ}$ ao $5^{\circ}$ ano do ensino fundamental de uma escola da rede básica estadual do município de Florianópolis. Como critérios de inclusão foram considerados: pertencer à faixa etária estabelecida (8 a 10 anos); apresentar intenção em participar voluntariamente do estudo; ter a autorização dos pais ou responsáveis por meio da assinatura do Termo de Consentimento Livre e Esclarecido (TCLE); não possuir problemas físicos ou deficiências cognitivas que impossibilitassem a realização das avaliaçóes, os quais seriam indicados previamente pela equipe pedagógica ou pelos pais. explique a relação entre os aspectos cognitivos e motor e que identifique qual bateria motora é mais indicada para tal investigação, ainda é limitada.

Com isso, acredita-se que, identificar qual bateria motora explica melhor às possíveis associaçóes entre as funções executivas e o desempenho motor, poderá auxiliar na compreensão dessa relação. Além do mais, torna-se necessário verificar se há relação destas variáveis em participantes com desenvolvimento típico, tendo em vista que grande parte dos estudos são realizados em crianças com comprometimento motor ${ }^{12-14}$. Dessa maneira, o objetivo deste estudo é verificar se há relação entre o desempenho motor e as funçóes executivas, bem como, identificar qual bateria motora, MABC-2, KTK, TGMD-2, explica melhor as funçóes executivas de crianças de 8 a 10 anos com desenvolvimento típico.

\section{Instrumentos e Procedimentos}

Para avaliação das funçôes executivas foi utilizado o teste de trilhas. Trata-se de um teste composto por duas partes (A e B). A parte A compreende dois momentos separadamente, um para letras e outro para números. No primeiro momento sáo apresentadas 12 letras (de "A" a "L"), dispostas de forma aleatória em uma folha, na qual o participante deve liga-los de acordo com a ordem alfabética, em um tempo de 1 minuto. No segundo momento da parte $\mathrm{A}$, são apresentados 12 números (de " 1 " a "12") dispostos de forma aleatória em uma folha, e o participante deve liga-los em conformidade com a ordem numérica, também com o tempo de 1 minuto. Por fim, na parte B, é apresentada uma sequência de 24 itens dispostos randomicamente numa folha. Os itens correspondem a 12 letras ("A" a "L") e 12 números ("1" a "12") conjuntamente, sendo que o respondente deve ligar os itens alternando a sequência alfabética e numérica (ex: "A-1-B-2"), num tempo de 1 minuto. Dentro das funçôes executivas, este instrumento avalia a percepção e atenção visual, a velocidade e o rastreamento visiomotor, a atenção sustentada e a velocidade de processamento e a flexibilidade cognitiva, sendo este último elemento exclusivo da parte $\mathrm{B}^{15}$. Os resultados são contabilizados a partir do número de sequências e itens corretamente ligados, bem como do escore bruto que é convertido em uma pontuação padrão de acordo com a idade ${ }^{16}$. A 
avaliação psicométrica do teste foi verificada por Frazen e Iverson ${ }^{17}$ e Charter et al. ${ }^{18}$ que observaram valores de confiabilidade oscilando de 0,78 a 0,92 na população investigada.

A avaliaçáo motora foi realizada por meio dos testes Movement Assessment Battery for Children Second Edition (MABC -2), Körperkoordination Test fürKinder (KTK) e Test of Gross Development Second Edition (TGMD-2), os quais serão descritos a seguir.

O MABC-2 é um teste motor que avalia três categorias de habilidades (Destreza Manual, Lançar e Receber e Equilíbrio), apropriadas para as faixas etárias específicas: Faixa um (3 a 6 anos); Faixa dois (7 a 10 anos) e Faixa três (11 a 16 anos). Para cada faixa etária são estabelecidas tarefas diferenciadas, de acordo com os diferentes níveis de complexidade. Neste estudo, especificamente, foi utilizada a faixa etária dois (7 a 10 anos). Após a aplicação da bateria, os escores brutos (tempo para realizar as tarefas ou quantidade de acertos) são convertidos em escores padrão, em que são somados dentro de cada categoria de habilidade. Por meio dessa pontuação, obtém-se o escore padrão total do teste e o resultado bruto total, os quais são comparados a uma tabela de percentis, que possibilitam classificar o desempenho motor das crianças. As crianças com resultado abaixo ou igual ao $5^{\circ}$ percentil, são consideradas com dificuldade no movimento, os valores entre o $5^{\circ}$ e o $15^{\circ}$ percentil indicam que a criança está com indicativo de risco para dificuldade de movimento e por fim os valores superiores ao $16^{\circ}$ percentil indicam que a criança não apresenta dificuldades de movimento ${ }^{19}$.

Este instrumento foi validado para população brasileira por VALENTini et al. ${ }^{20}$. Esse processo de validação envolveu 13 especialistas em Desenvolvimento Motor e um total de 844 crianças (3 a 13 anos) de dois estados diferentes no Brasil. O teste foi rigorosamente testado quanto à sua confiabilidade e validade de face, conteúdo, critério e construto, mostrando-se valido para população brasileira.

Foi encontrada uma alta confiabilidade intra e inter avaliadores $(r=0,74 ; \mathrm{p}<0,0001)$ e consistência interna para o MABC-2 foi estabelecida para crianças brasileiras $(\alpha=0,78)$. Além disso, na análise discriminante confirmou o poder MABC-2 (.80) para diferenciar crianças com DCD e aquelas em risco de DCD de crianças com desenvolvimento típico.

O KTK foi desenvolvido por Kiphard e
SCHILling ${ }^{19}$. Ele consiste em investigar o nível de coordenação motora de crianças e adolescentes de cinco a 14 anos de idade. Este teste avalia a coordenação motora, por meio das capacidades motoras: coordenação corporal, equilíbrio, velocidade e força, distribuídas em quatro tarefas motoras, (1) trave de equilíbrio; (2) saltitar com uma perna; (3) saltos laterais; e (4) transposição lateral. As pontuaçóes de cada tarefa são convertidas em escores padronizados, de acordo com o sexo e a idade de cada criança. Esses escores são somados resultando no Quociente motor (QM), o qual permite classificar o desempenho das crianças em cinco categorias: perturbaçóes da coordenação (quociente motor $\leq 70$ pontos); insuficiência coordenativa (quociente motor $\leq 71$ a 85 pontos); (3) coordenação normal (quociente motor $\leq 86$ a 115 pontos); (4) coordenação boa (quociente motor $\leq 116$ a 130 pontos); (5) coordenação muito boa (quociente motor $\leq 131$ a 145 pontos).

As características psicométricas do KTK foram testadas por Kiphard e SCHILling ${ }^{19}$ em 1228 crianças alemãs, sendo encontrados valores de coeficiente de fidedignidade de 0.97 para os escores brutos e 0.80-0.96 para os testes individuais. A análise fatorial dos testes individuais resultou em um único fator que apresentou um percentual de variância explicado pelos quatro testes de $81 \%$ para os seis anos e 98\% para os nove anos. Além do mais, foram observados valores de $\mathrm{r}$ que variaram de 0.60 a 0.81 indicando correlaçóes entre os quatro testes. Não foram verificados estudos de validação para a população brasileira.

O TGMD-2 consiste em uma avaliação normativa das habilidades motoras globais consideradas comuns. É composto por doze habilidades motoras fundamentais, divididas em dois subtestes específicos: habilidades de locomoção (correr, galopar, saltitar, saltar obstáculo, saltar horizontalmente e deslizar); e habilidades de controle de objetos (rebater, quicar, receber, chutar, arremessar por cima do ombro e rolar a bola). Para cada habilidade são observados entre 3 a 5 critérios motores específicos que permitem avaliar como as crianças coordenam o tronco e os membros durante o desempenho de uma habilidade motora, ou seja, analisa o padrão da habilidade motora, a qual é executada duas vezes, sendo as duas avaliadas e pontuadas. Os subtestes possuem 24 critérios de desempenho cada um, sendo atribuída uma nota 0 (não atendeu ao critério) ou 1 (atendeu 
ao critério), totalizando um máximo de até 2 pontos por critério em cada habilidade. Desta forma, cada criança avaliada pode ter no máximo 48 pontos no escore de cada subteste. A partir desta pontuação é atribuído o quociente motor bruto e as classificaçôes "muito pobre", "pobre", "abaixo da média", "na média", "acima da média", "superior" e "muito superior" de acordo com o sexo e a faixa etária. Para a execuçáo deste teste, utilizou-se uma câmara filmadora, conforme protocolo estabelecido pelo autor do teste, três avaliadores de forma independente previamente trinados e capacitados foram responsáveis pelas avaliaçóes do desempenho motor de todas as crianças a partir dos vídeos individuais.

Este instrumento foi validado para a populaçáo brasileira por VALENTini et al. ${ }^{21}$ que ao investigar 587 crianças de Porto Alegre RS obtiveram índices satisfatórios de validade fatorial confirmatória, com uma razão entre o Qui-quadrado e graus de liberdade de 3.38; Goodness-of-fitIndex de 0.95; Ajusted Goodnessof-fit index de 0.92 e Tucker e Lewis's Index of Fit de 0.83; além do mais, evidência de correlação entre o teste e o reteste para todos os subtestes foram verificadas, sendo altamente significativa e forte para a locomoção $(\mathrm{r}=0.82$, $\mathrm{p}$-valor $<0.01)$ e controle de objetos $(\mathrm{r}=0.88$, $\mathrm{p}$-valor $<0.01)$.

Inicialmente, após a aprovação do comitê de ética e autorização do dirigente da instituição de ensino para a realização da pesquisa, as crianças foram contatadas e convidadas a participar da pesquisa mediante a entrega do TCLE assinado pelos pais ou responsáveis. Em posse deste documento, a coleta de dados, que ocorreu em dois momentos foi iniciada. Primeiramente foram coletados os dados referentes aos aspectos cognitivos, à aplicaçáo dos mesmos foi realizada de forma coletiva, ou seja, todos os participantes realizavam os testes num mesmo momento, após as orientaçóes do pesquisador responsável

\section{Resultados}

Na TABELA 1 estão expostos os valores de média e desvio padrão obtidos nos testes de avaliaçáo da função executiva e da atenção concentrada. Pode-se observar que de forma geral os meninos apresentaram valores superiores em relação às meninas para todas as variáveis investigadas, pela aplicação e o preenchimento de um teste simplificado, realizado a fim de familiarizar os participantes com os testes. Todas estas coletas foram acompanhadas por dois avaliadores, um responsável pelas orientaçóes e pelo controle do tempo e outro em verificar e controlar para que todos finalizassem o preenchimento ao comando final do teste.

Posteriormente aos testes cognitivos foram realizadas as avaliaçóes motoras. As três baterias motoras foram aplicadas em dias diferentes de forma individual, por avaliadores treinados em cada bateria que era responsável, em um espaço adequado que permitisse a realização das avaliaçóes sem a ocorrência de interferências ou riscos aos participantes durante a execução. Ressaltando que a aplicaçáo dos testes foi executada de acordo com os protocolos de execuçáo de cada bateria motora por pesquisadores previamente treinados.

\section{Análise Estatística}

Os dados foram tabulados e analisados no programa estatístico Statistical Package for Social Sciences (SPSS) for Windows versão 20.0. Recorreuse a análise descritiva das variáveis por meio de indicadores estatísticos de tendência central (média), variabilidade (desvio padráo) e frequências relativa (\%) e absoluta (n). A fim de verificar a distribuição dos dados foi utilizado o teste de normalidade de Kolmogorov-Smirnov. A comparação entre os valores obtidos com os testes executivos e motores de acordo com o sexo foi analisada pelo teste $U$ de Mann Whitney, uma vez que os dados não apresentaram distribuição normal. A relação entre os valores brutos das funçóes executivas foi verificada por meio do teste de Correlaçẫo de Spearman. Por fim, utilizou-se a Regressão linear simples para identificar quais os testes motores (MABC-2, KTK e TGMD-2) melhor explicam as funçôes executivas. Para todas as análises foi considerado um $\mathrm{p}$-valor $<0.05$. porém estas diferenças entre os sexos não foram estatisticamente significativas ( $\mathrm{p}$-valor $>0,05$ ).

No que se refere à avaliação do desempenho motor das crianças investigadas. As meninas apresentaram valores de média melhores que os meninos para as baterias MABC-2 e KTK, enquanto os 
meninos demonstraram melhores resultados no TGMD-2. Porém, estas diferenças não foram estatisticamente significativas (p-valor $>0,05$ ) (TABELA 2).

TABELA 1 - Valores demédia e desvio padrão do desempenho cognitivo (função executiva eatenção concentrada) de escolares, de acordo com sexo. Florianópolis, Santa Catarina, Brasil, 2015.

\begin{tabular}{lcccc}
\hline \multirow{2}{*}{ Variável } & Masculino & Feminino & Geral & \multirow{2}{*}{ p-valor } \\
\cline { 2 - 4 } & Média (DP) & Média (DP) & Média (DP) & \\
\hline Funçáo Executiva & & & & \\
TTA & $83,7(42,4)$ & $76.0(46.3)$ & $79.8(44.2)$ & 0.55 \\
TTB & $93,5(46,4)$ & $82.7(51.8)$ & $88.1(49.1)$ & 0.72 \\
\hline
\end{tabular}

DP: Desvio padrão; TTA: Teste de Trilhas parteA; TTB: Teste de Trilhas parte $B$; * $p<0.05$ testeUdeMann Whitney.

TABELA 2 - Valores de média e desvio padrão do desempenho motor (escores gerais das baterias motoras, MABC2, KTK e TGMD-2) de escolares de acordo com o sexo. Florianópolis, Santa Catarina, Brasil, 2015.

\begin{tabular}{lcccc}
\hline \multirow{2}{*}{ Variável } & Masculino & Feminino & Geral & \multirow{2}{*}{ p-valor } \\
\cline { 2 - 4 } & Média (DP) & Média (DP) & Média (DP) & \\
\hline MABC-2 & $67.3(17.5)$ & $68.4(8,6)$ & $67.8(13.8)$ & 0.59 \\
TGMD-2 & $95.0(14.2)$ & $79.9(10.4)$ & $78.2(9.6)$ & 0.33 \\
KTK & $76.5(8.6)$ & $91.7(17.1)$ & $93.4(15.6)$ & 0.12 \\
\hline
\end{tabular}

DP: Desvio padrão; MABC-2: Movement Assessment Battery for Children;

TGMD-2: Test of Gross Development Second Edition; KTK: KörperkoordinationstestFür Kinder. ${ }^{*} \mathrm{p}<0.05$ teste $U$ de Mann-Whitney.

Ao correlacionar os escores gerais das baterias motoras com as variáveis cognitivas, pode-se identificar que somente o MABC-2 apresentou uma correlação moderada com a função executiva para o sexo feminino, sendo esta positiva, ou seja, as meninas com melhor desempenho nos testes de função executiva (TTA e TTB) foram as que tiveram melhor desempenho no teste motor $(r=0.39$; p-valor $<0.05)$ e $(r=0.43$; p-valor $<0.05)$, respectivamente.

Para o sexo masculino, o MABC-2 só esteve correlacionado de maneira moderada com o TTA, de forma que os meninos que apresentaram escores superiores no teste motor foram os que demonstraram melhor desempenho para a percepção e atenção visual, a velocidade o rastreamento visomotor, especialmente $(r=0.47$; p-valor<0.05) (TABELA 3).

De forma geral, ao realizar a regressão linear simples entre os escores dos testes motores com o TTA e o TTB. O teste motor MABC-2 foi o único que explicou funçóes executivas, TTA $\left(\mathrm{r}^{2}\right.$ $=0.15 ; \beta=0.07 ; \mathrm{p}<0.05)$ e TTB $\left(\mathrm{r}^{2}=0.19 ; \beta=\right.$ $0.07 ; \mathrm{p}<0.05)$ para o sexo feminino, mesmo com um poder de explicação baixo, cada aumento de uma unidade nos escores do MABC-2 o nível de TTA total aumenta 0.15 e o de TTB 0.19. Nos meninos, o MABC-2 explicou somente a variável TTA $\left(r^{2}=0.08 ; \beta=0.05 ; p<0.05\right)$, demonstrando que para cada acréscimo de uma unidade no escore do teste motor a pontuação no teste cognitivo TTA aumenta 0.8 (TABELA 4). 
$r$ : Coeficiente de correlação;

TTA: Teste de Trilhas parte A;

TTB: Teste de Trilhas parte B;

MABC-2: Movemen-

tAssessmentBattery for Children;

TGMD-2: Test of Gross

DevelopmentSecon-

dEdition;

KTK: KörperkoordinationstestFürKinder.

* $p<0.05$ correlação de Spearman.
$R^{2}$ : coeficiente de regressão;

TTA: Teste de Trilhas parte $A$;

TTB: Teste de Trilhas parte $B$;

MABC-2: Movement Assessment Battery for Children;

TGMD-2: Test of Gross Development Second

Edition;

KTK:

Körperkoordinations test Für Kinder. ${ }^{*} p<$ 0.05 Regressão linear simples.

TABELA 3 - Correlação entre função cognitiva e os escores gerais dos testes motores de escolares. Florianópolis, Santa Catarina, Brasil, 2015.

\begin{tabular}{lcc}
\hline & \multicolumn{2}{c}{ Sexo feminino } \\
\cline { 2 - 3 } & TTA & TTB \\
\cline { 2 - 3 } MABC-2 & R & $\mathbf{r}$ \\
TGMD-2 & $0.39^{*}$ & $0.43^{*}$ \\
KTK & -0.18 & 0.13 \\
\hline & 0.43 & 0.60 \\
\cline { 2 - 3 } & & Sexo Masculino \\
\hline MABC-2 & TTA & TTB \\
TGMD-2 & $\mathbf{R}$ & -0.08 \\
KTK & $0.47^{*}$ & 0.04 \\
\hline
\end{tabular}

TABELA 4 - Regressão linear simples entre os escores brutos do desempenho cognitivo com os escores gerais dos testes motores de escolares. Florianópolis, Santa Catarina, Brasil, 2015.

\begin{tabular}{|c|c|c|}
\hline & \multicolumn{2}{|c|}{ Sexo feminino } \\
\hline & TTA & TTB \\
\hline & $\mathbf{R}^{2}$ & $\mathbf{r}^{2}$ \\
\hline MABC-2 & $0.15^{*}$ & $0.19^{*}$ \\
\hline TGMD-2 & 0.03 & 0.03 \\
\hline \multirow[t]{4}{*}{ KTK } & 0.00 & 0.001 \\
\hline & \multicolumn{2}{|c|}{ Sexo Masculino } \\
\hline & TTA & TTB \\
\hline & $\mathbf{R}^{2}$ & $\mathbf{r}^{2}$ \\
\hline MABC-2 & $0.08^{*}$ & 0.06 \\
\hline TGMD-2 & 0.04 & 0.03 \\
\hline KTK & 0.008 & 0.000 \\
\hline
\end{tabular}

\section{Discussão}

O objetivo deste estudo foi verificar se há relação entre o desempenho motor e as funçôes executivas, bem como identificar qual bateria motora, MABC-2, KTK, TGMD-2, explica melhor as funçóes executivas de crianças de 8 a 10 anos com desenvolvimento típico. Dentre os principais resultados, destaca-se que, não se encontrou diferença significativa entre meninos e meninas no que diz respeito à função executiva, da mesma forma acontece no que se refere ao desempenho motor avaliado pelas baterias MABC-2, TGMD2 e KTK. Ademais, verificou-se que apenas a 
bateria motora MABC-2 apresentou correlação com o teste de função executiva. Para o sexo feminino correlacionou-se com as partes TTA e TTB, já para o sexo masculino, correlacionou-se apenas com os escores do TTB. Ao investigar quais testes motores explicam melhor as funçóes executivas, encontrou-se que o MABC-2 foi o único que explicou as funções executivas, totalmente para o sexo feminino e parcialmente para o sexo masculino.

No que se refere à função executiva de meninos e meninas, pesquisas demonstram que a maioria dos parâmetros do modelo estrutural das funçôes executivas são invariantes para o sexo e não diferem em termos de inibição e flexibilidade quando diz respeito ao sexo ${ }^{22,23}$. Esses resultados vão ao encontro da presente investigação e do estudo de Wiebe et al. ${ }^{24}$, que apontam que meninos e meninas não se diferenciaram em termos de capacidade de função executiva na idade pré-escolar.

Contudo, outras pesquisas indicam que a relação entre as funçóes executivas e o sexo ainda não estão bem definidos, visto que resultados anteriores demonstram que meninos e meninas diferiram quanto à capacidade de atenção e atenção visual, sendo que as meninas apresentaram melhores resultados ${ }^{25,26}$. A possível explicação para essa incongruência na literatura, pode estar nas variáveis que não são controladas nos estudos, como atitudes dos pais, ambiente doméstico e status socioeconômico, visto que a qualidade do ambiente familiar por meio da sensibilidade materna, estimulação cognitiva e recursos disponíveis, pode impulsionar o desenvolvimento de determinados aspectos das funções executivas em algumas faixas etárias específicas ${ }^{27,28}$.

Já em relação ao desempenho motor, os resultados da presente pesquisa vão ao encontro da literatura, na qual evidencia pouca diferença entre os sexos no que diz respeito a essa variável tanto em crianças com desenvolvimento típico ${ }^{29-31}$. Teóricos do desenvolvimento humano e motor ressaltam que as diferenças entre os sexos estão relacionadas às expectativas pessoais e sociais vivenciadas pela criança desde o seu nascimento, as quais podem direcionar seus comportamentos $^{30,32}$.

Com isso, ressalta-se que a não diferenciação entre os participantes do presente estudo no que tange o desempenho motor, podem ser justificados pelo contexto sociocultural que os participantes estão inseridos. Esses no qual, aliados às condiçôes socioeconômicas e as características da família, tanto os meninos quanto as meninas, possuem as mesmas oportunidades motoras e acabam por vivenciar as mesmas experiências, e dessa forma apresentam-se com desempenho motor homogêneo, não se diferenciando em relação aos sexos.

No que diz respeito às associaçóes entre a função executiva e o desempenho motor, verificou-se que as meninas que obtiveram melhor desempenho nas funções executivas tanto no TTA, como no TTB, também apresentaram melhor desempenho motor na bateria MABC2. Já para o sexo masculino, os meninos que apresentaram escores superiores no MABC-2, também apresentaram melhor desempenho apenas no teste cognitivo TTA. A partir de uma perspectiva neuropsicológica estudos indicam que, existem explicaçóes para a coocorrência de desempenho motor e das funçóes executivas, tendo em vista que as funções motoras e cognitivas são acopladas por meio do uso das mesmas estruturas cerebrais ${ }^{6}$, por exemplo, o cerebelo está envolvido em funçóes motoras e cognitivas e o córtex pré-frontal desempenha um papel importante no funcionamento cognitivo, bem como no desempenho motor através das fortes conexóes neurais. As técnicas de neuroimagem mostram que as regióes importantes para o desempenho motor e cognitivo, como o cerebelo, o córtex pré-frontal e as estruturas de conexão (incluindo os gânglios basais), são coativadas durante tarefas motoras e cognitivas ${ }^{33,34}$ o que explica a associação entre as variáveis encontradas no presente estudo.

Ademais, ao investigar quais testes motores poderiam explicar melhor as funçóes executivas, encontrou-se que o MABC-2 foi à única bateria que explicou as funçôes executivas avaliadas, totalmente para o sexo feminino e parcialmente para o sexo masculino. Neste sentido, especificamente esse resultado pode se dar devido ao fato de que a bateria motora MABC-2, apresenta-se como um teste com maior sensibilidade para apurar tarefas relacionadas às habilidades motoras finas, como às tarefas das dimensóes de destreza manual e lançar e receber que possivelmente estão diretamente relacionadas com processos cerebrais mais complexos ${ }^{7}$, sendo o teste que possivelmente mais se aproxime das funçóes executivas.

Tais dimensôes, exigem em maior proporção aspectos como tomada de decisão, capacidade de 
planejamento, percepçáo e atenção visual, quando comparados a execução de ações provenientes de outras baterias motoras como, o TGMD-2 e o KTK, uma vez que essas são compostas por habilidades motoras mais amplas e supóem-se que não exigem os mesmos aspectos cognitivos que envolvem às funçôes executivas como nas tarefas presentes na bateria MABC-2.

Além disso, pode-se justificar o resultado apenas para o sexo feminino, principalmente quando se considera que as meninas possuem práticas motoras que envolvem predominantemente habilidades motoras finas e de destreza manual, o que muitas vezes pode ser determinado pelas experiências da infância ou por preferência, onde são mais expostas a atividades que envolvam maior precisão de movimento, nas quais as habilidades manipulativas são mais exigidas.

Dentre as limitações dessa investigação estão o não controle de variáveis intervenientes como o envolvimento com tarefas extraescolares e influência dos pais e familiares. Em relação aos pontos positivos, destacam-se as características socioeconômicas homogêneas dos participantes e crianças com desempenho motor típico, visto que a maior parte dos estudos são conduzidos com crianças com Desordem
Coordenativa Desenvolvimental. Além do mais, sugere-se que em futuros estudos sejam observadas questôes referentes a diferentes faixas etárias, para averiguar o comportamento dessa relação ao longo do crescimento e desenvolvimento do indivíduo.

Dessa maneira conclui-se que, embora o teste motor MABC-2 seja uma ferramenta mundialmente reconhecida para averiguação de dificuldades significativas de movimento, os resultados aqui encontrados, implicam que ao utilizar o referido teste, além de uma detecção das dificuldades motoras, é possível realizar uma detecção prévia parcial de possíveis déficits nas funções executivas para as meninas. Ou seja, ao apresentar um percentil baixo no MABC-2, sugerese investigar náo apenas o desempenho motor, mas também as funçóes executivas com testes adequados para a avaliação das mesmas.

Com isso, aconselha-se para os profissionais da área a utilizaçáo da bateria MABC-2 para pesquisas que tenham como intuito a investigação da relação entre as funções executivas e o desempenho motor. Ao mesmo tempo, com esses resultados, presume-se que apenas algumas tarefas motoras estão relacionadas à função executiva, sendo aquelas que envolvem habilidades motoras finas.

\section{Abstract}

The motor tests MABC-2, TGMD-2 and KTK can explain the executive function in children of 8 to 10 years?

The aim of this study was to verify if there is a relationship between motor performance and executive functions, as well as, to analyze which motor battery, MABC-2, KTK, TGMD-2, would better explain the executive functions of 8- to 10-year-old children with development typical. 60 children, aged between 8 and 10 years (Mean $=9.09$; Standard deviation 0.75 ) were included in the study. For the evaluation of the executive functions, the Test of Trails was used, already for the evaluation of the motor performance, the tests Movement Assessment Battery for Children Second Edition (MABC -2), Körperkoordination Test fürKinder (KTK) and Test of Gross Development Second Edition (TGMD-2). The Spearman Correlation test was used to verify the relationship between executive functions and motor performance, and Simple Linear Regression was used to identify which motor tests best explain executive functions. We found a relationship between motor performance and executive function only for girls. In addition, it has been found that the MABC-2 battery is the test that best explains partially the executive functions mainly for the female gender. These results imply a possible prior detection of both motor and cognitive difficulties, using the MABC-2 battery for females in this group. Furthermore, with these results, it is assumed that only some motor tasks are related to the executive function, being those involving fine motor skills.

KEYwoRds: Motor Skill; Executive control; Motor batteries; Typical development. 


\section{Referências}

1. Wu, Meng et al. Infant motor and cognitive abilities and subsequent executive function. Infant Behavior Develop. 2017;49: 204-213.

2. Diamond A. Executive functions. Ann Rev Psychol. 2013;64:135-168.

3. Malloy-Diniz LF, Sedo M, Fuentes D, Leite WB. Neuropsicologia das funçôes executivas. In: Fuentes D, Malloy-Diniz LF, Camargo CHP, Cosenza RM, editores. Neuropsicologia: teoria e prática. Porto Alegre: Artmed; 2008.

4. Claire EC, Laura LB, William MM. Fine motor skills and executive function both contribute to kindergarten achievement. Child Dev. 2012;83:1229-1244.

5. Roebers CM, Kauer M. Motor and cognitive control in a normative sample of 7-year-olds. Develop Sci. 2009;14: 175-181.

6. Diamond, A. Close interrelation of motor development and cognitive development and of the cerebellum and prefrontal cortex. Child Develop. 2000;71:44-56.

7. Van der Fels SC, Wierike EH, Elferink-Gemser, JC. Visscherthe relationship between motor skills and cognitive skills in 4-16 year old typically developing children: a systematic review. J Sci Med Sport. 2015;18:697-703.

8. Wang MV, Lekhal R, Aarø LE, et al. Developmental relationship between language and motor performance from 3 to 5 years of age: a prospective longitudinal population study BMC Psychol. 2014;2:34.

9. Ten Eycke D, Dewey KD. Parent-report and performance-based measures of executive function assess different constructs Child Neuropsychol. 2015.

10. Houwen S, et al. The relationship between motor performance and parent-rated executive functioning in 3-to 5-yearold children: what is the role of confounding variables?. Human Mov Sci. 2017;53:24-36.

11. Hernandez AM, Caçola P. Motor proficiency predicts cognitive ability in four-year-olds. Eur Early Childhood Educ Res J. 2015;23:573-584.

12. Livesey D, et al. The relationship between measures of executive function, motor performance and externalising behaviour in 5-and 6-year-old children. Human Mov Sci. 2006;25:50-64.

13. Rigoli D, et al. An examination of the relationship between motor coordination and executive functions in adolescents. Develop Med Child Neurol. 2012;54:1025-1031.

14. Piek JP, et al. The relationship between motor coordination, executive functioning and attention in school aged children. Arch Clin Neuropsychol. 2004;19:1063-1076.

15. Ble A, et al. Executive function correlates with walking speed in older persons: the inchianti study. J Am Geriatrics Soc. 2005;53:410-415.

16. Dias N, Tortella G. Evidências de validade do teste e trilhas: partes A e B. In: Seabra A. Avaliaçáo neuropsicológica cognitiva: atenção e funçóes executivas. São Paulo: Memnon. 2012;67-72.

17. Frazen MD, Paul D, Iverson GL. Cross-validation of the alternate forms reliability of the Trail Making Test. Clin Neuropsychologist. 1996;10:125-129.

18. Charter RA, Adkins TG, Alekoumbides A, Seacat GF. Reliability of the WAIS, WMS, and Reitan Battery: raw scores and standardized scores corrected for age and education. Int J Clin Neuropsychol. 1987;9:28-32.

19. Kiphard EJ, Schilling, F. Körperkoordinationstest für Kinder: KTK. Beltz.1974.

20. Valentini NC, Ramalho MH, Oliveira MA. Movement assessment battery for children-2: translation, reliability, and validity for Brazilian children. Res Develop Disabilities. 2014;35:733-740.

21. Valentini ND, et al. Teste de desenvolvimento motor grosso: validade e consistência interna para uma população gaúcha. Rev Bras Cineantropometria Des Hum. 2008;10:399-404.

22. Ferreira LO, et al. Avaliação da função executiva de crianças e adolescentes: desempenho e tempo na Torre de Hanói. 2013.

23. Monette S, Bigras M, Lafrenière MA. Structure of executive functions in typically developing kindergarteners. J Exp Child Psychol. 2015;140:120-139.

24. Wiebe SA, Sheffield T, Nelson JM, et al. The structure of executive function in 3- year-olds. J Exp Child Psychol. 2011;108:436-452.

25. Klenberg L, Korkman M, Lahti-Nuuttila P. Differential development of attention and executive functions in 3- to 12- year-old Finnish children. Develop Neuropsychol. 2001;20:407-428.

26. Silverman IW. Gender differences in delay of gratification: a meta-analysis. Sex Roles. 2003;49:451-463.

27. Papalia DE, Feldman RD. Desenvolvimento humano. Artmed Editora; 2013.

28. Sbicigo JB, et al. Nível socioeconômico e funções executivas em crianças/ adolescentes: revisão sistemática. Arq Bras 
Fronza FC, et al.

Psicol. 2013;65:51-69.

29. Medeiros P, Zequinão MA, Cardoso FL. A influência do desempenho motor no "status" social percebido por crianças. Rev Bras Educ Fís Esporte. 2016;30:1069-1077.

30. Lung FW, Chiang TL, Lin SJ, et al. Gender differences of children's developmental trajectory from 6 to 60 months in the Taiwan birth cohort pilot study. Res Develop Dis. 2011;32:100-106.

31. Saccani R., Valentini NC. Análise do desenvolvimento motor de crianças de 0 a 18 meses de idade: representatividade dos itens da Alberta Infant Motor Scale por faixa etária e postura. Rev Bras Cresc Des Hum. 2010;20:753-764.

32. Haywood KM, Getchell N. Desenvolvimento motor ao longo da vida. Porto Alegre: Artmed; 2010.

33. Howard AD, Okely YG. Ellisevaluation of a differentiation model of preschoolers' executive functions. Frontiers Psychol. 2015;6:285.

34. Piek B, Hands MK. Licariassessment of motor functioning in the preschool period. Neuropsychol Rev. 2012;22:402-413.

\section{ENDEREÇO}

Pâmella de Medeiros

Rua Pascoal Simone, 358 - Coqueiros 88080-350 - Florianópolis - SC - Brasil E-mail: pamellademedeiros@hotmail.com
Submetido: 30/01/2018

Revisado: 17/05/2018

Aceito: 20/05/2021 